\title{
On Higher Eta-Invariants and Metrics of Positive Scalar Curvature
}

\author{
ERIC LEICHTNAM ${ }^{1, \star}$ and PAOLO PIAZZA ${ }^{2, \star \star ~}$ \\ ${ }^{1}$ Institut de Jussieu-Chevaleret, Plateau E, $7^{\text {me }}$ étage (Algèbres d'opérateurs), 175 rue de \\ Chevaleret, 75013 Paris, France.e-mail: leicht@math.jussieu.fr \\ ${ }^{2}$ Università di Roma 'La Sapienza', Dipartimento di Matematica 'Guido Castelnuovo', \\ P.le A. Moro 2, I-00185 Roma, Italy.e-mail: piazza@mat.uniromal.it
}

(Received: June 2001)

\begin{abstract}
Let $N$ be a closed connected spin manifold admitting one metric of positive scalar curvature. In this paper we use the higher eta-invariant associated to the Dirac operator on $N$ in order to distinguish metrics of positive scalar curvature on $N$. In particular, we give sufficient conditions, involving $\pi_{1}(N)$ and $\operatorname{dim} N$, for $N$ to admit an infinite number of metrics of positive scalar curvature that are nonbordant.
\end{abstract}

Mathematics Subject Classifications (2000): 55N22, 19L41.

Key words: bordism groups, positive scalar curvature metrics, Galois coverings, higher eta-invariants, higher $\rho$-invariants, $b$-pseudodifferential calculus, higher APS index formula.

\section{Introduction}

Let $N^{n}$ be a closed connected spin manifold of dimension $n$. In this Introduction we shall always assume that $N$ admits at least one metric of positive scalar curvature $g$. One may ask how many such metrics there are on $N$. As a small perturbation of $g$ produces a different metric which is still of positive scalar curvature (三PSC), it is clear that in order to give a rigorous meaning to the above question one first needs to introduce a way for distinguishing two metrics of PSC $g_{0}, g_{1}$. There are three increasingly stronger conditions for distinguishing two metrics of PSC $g_{0}$ and $g_{1}$.

The first one is to say that $g_{0}$ and $g_{1}$ are not path-connected in $\mathcal{R}^{+}(N)$, the space of PSC metrics on $N$. Thus, in this case, we are interested in $\pi_{0}\left(\mathcal{R}^{+}(N)\right)$, the set of arcwise connected components of $\mathcal{R}^{+}(N)$.

The second way for distinguishing two PSC metrics employs the notion of concordance: $g_{0}$ and $g_{1}$ are concordant if there exists a metric of PSC on $N \times[0,1]$ extending $g_{0}$ on $N \times\{0\}, g_{1}$ on $N \times\{1\}$ and of product-type near the boundary. Notice that two metrics which are path connected are concordant; it is not known

\footnotetext{
${ }^{\star}$ Research partially supported by a CNR-CNRS cooperation project.

${ }^{\star \star}$ Research partially supported by a CNR-CNRS cooperation project and by MURST.
} 
whether the converse is true. In this case one is interested in the set of concordance classes of PSC metrics on $N$, denoted by $\tilde{\pi}_{0}\left(\mathcal{R}^{+}(N)\right)$.

The third and more subtle way for distinguishing two PSC metrics $g_{0}, g_{1}$ on our spin manifold $N$ employs the notion of bordism. Intuitively we allow ourself the freedom of taking any spin manifold $W$ with boundary $\partial W=N \amalg-N$ and a PSC metric $G$ on $W$ which is of product-type near the boundary and restricts to $g_{0} \bigsqcup g_{1}$ on $\partial W$. See Section 1 for the precise definition. Clearly, two metrics that are concordant are also bordant. (On the other hand there are example of nonconcordant metrics that are bordant, see [LM, p. 329].) Summarizing, as far as the problem of distinguishing metrics of positive scalar curvature is concerned, we have: nonbordant $\Rightarrow$ nonconcordant $\Rightarrow$ nonpathconnected. In this paper we shall be mainly concerned with nonbordant metrics.

Interesting results by Gromov and Lawson [GL], Lawson and Michelson [LM], Kreck and Stolz [KS], Botvinnik and Gilkey [Bo-Gi] and others show that, under some assumptions on $N$, if $N$ admits one metric of PSC then $N$ admits an infinite number of 'distinct' metrics of PSC. It is important to remark that all these papers use index theory for Dirac operators in an essential way; the main point is to define invariants that can distinguish two metrics of PSC. In the case of [GL, LM, Bo-Gi] and $[\mathrm{KS}]$ these invariants involve, one way or another, the Atiyah-Patodi-Singer index theory on manifolds with boundary. Of particular importance for us is the paper by Botvinnik and Gilkey [Bo-Gi]. The fundamental idea used in [Bo-Gi] is that the twisted eta-invariant is a bordism invariant and can, therefore, be used in order to distinguish non-bordant metrics; using the twisted eta-invariants together with some deep facts about PSC metrics, Botvinnik and Gilkey are able to give sufficient conditions on $\pi_{1}(N)$ and $\operatorname{dim} N$ ensuring the existence of an infinite number of nonbordant metrics.

Our main goal in this paper is to give an extension of the Botvinnik-Gilkey's result by replacing the twisted eta-invariant used in [Bo-Gi] by Lott's higher etainvariant $[\mathrm{L} 2,3]$ and its delocalized part, which is, by definition, the higher $\rho$ invariant. That the higher $\rho$-invariant could be used in connection with PSC problems was already observed in [L2], where it was proved that it is constant on the connected components of $\mathcal{R}^{+}(N)$. In this paper we elaborate on these ideas, relying heavily on the arguments given in [Bo-Gi]; the higher Atiyah-PatodiSinger index theory developed in [L2] and [LP1,3] will play an essential role throughout.

Although we shall give results that might be amenable to further applications, we prefer to state here the geometric outcome of our arguments. Let $F$ be a finite group and let $m$ be a odd positive integer. Botvinnik and Gilkey have introduced a nonnegative integer $r_{m}(F)$, see [Bo-Gi]. We are interested in finite groups $F$ and positive integers $m$ such that $r_{m}(F)>0$. In this direction we recall that if $m \equiv 3 \bmod 4$ or if $|F|$ is odd, then, automatically, $r_{m}(F)>0$. If $m \equiv 1 \bmod 4$ then $r_{m}(F)>0$ iff $F$ contains an element, which is not conjugate to its inverse. 
THEOREM 0.1. Let $n \in \mathbb{N}, n \geqslant 5$. Let $F$ be a finite group such that $r_{m}(F)>0$ for $m$ odd, $5 \leqslant m \leqslant n$. Let $\Gamma, \Gamma^{\prime}, \Delta$ be finitely generated groups. On these groups we make the following assumptions: (i) $\Gamma=\Gamma^{\prime}>\triangleleft F$; (ii) $\Delta$ is virtually nilpotent or Gromov hyperbolic; (iii) there exists $i \in \mathbb{N}$ such that $H^{n-m-4 i}(\Delta, \mathbb{Q}) \neq 0$. Let now $N$ be a closed connected spin manifold of dimension $n$ with fundamental group $\Gamma \times \Delta$ admitting one metric of positive scalar curvature. Then, $N$ admits an infinite number of nonbordant metrics of PSC.

\section{Spin Bordism Groups}

In this Section we recall the definition of the bordism groups that will be used in the sequel. Metrics on manifolds with boundary will be understood to be of product-type near the boundary; we make this assumption throughout the paper.

Let $\Gamma$ be a finitely generated group. Let $B \Gamma$ be the associated classifying space and let $E \Gamma \rightarrow B \Gamma$ be the universal $\Gamma$-covering. We denote by $\Omega_{n}^{\text {spin }}(B \Gamma)$ the bordism group consisting of triples $(N, s, f)$ with $N$ a spin $n$-dimensional closed compact manifold, $s$ a spin structure on $N$ and $f$ a continuous map $N \rightarrow B \Gamma$. We let $\Omega_{n}^{\text {spin, }+}(B \Gamma)$ be the subgroup of $\Omega_{n}^{\text {spin }}(B \Gamma)$ consisting of triples $(N, s, f)$ with $N$ admitting a metric of positive scalar curvature.

Finally, let $\operatorname{Pos}_{n}^{\text {spin }}(B \Gamma)$ be the bordism group consisting of quadruples $(N, s$, $f, g)$ with $g$ a metric of PSC on $N$. Two quadruples $(N, s, f, g)$ and $\left(N^{\prime}, s^{\prime}, f^{\prime}, g^{\prime}\right)$ are bordant in $\operatorname{Pos}_{n}^{\text {spin }}(B \Gamma)$ if there exists a spin $(n+1)$-dimensional manifold with boundary $W$ with a continuous map $F: W \rightarrow B \Gamma$, a spin structure $S$ and a positive scalar curvature metric $H$ on $W$ such that (with obvious notation) $\partial W=$ $N \bigsqcup\left(-N^{\prime}\right),\left.F\right|_{\partial W}=f \amalg f^{\prime}, S_{\partial W}=s \bigsqcup s^{\prime},\left.H\right|_{\partial W}=g \amalg g^{\prime}$.

DEFINITION 1.1. Let $N$ be a closed spin manifold of dimension $n$ admitting one metric of PSC. Let $f: N \rightarrow B \pi_{1}(N)$ be the classifying map for the universal cover of $N$. We shall say that two metrics of PSC $g_{0}$ and $g_{1}$ are bordant if for any spin structure $s$ we have: $\left[N, s, f, g_{0}\right]=\left[N, s, f, g_{1}\right]$ in $\operatorname{Pos}_{n}^{\text {spin }}\left(B \pi_{1}(N)\right)$.

We now pass to the relative bordism groups. These were first introduced by Hajduk [Ha]. They were successfully employed by Stolz in problems of classification of PSC metrics. See [St1] (p. 630) [St2]. Following [St1], we denote them by $R_{n}^{\text {spin }}(B \Gamma)$. Thus, we only consider the spin case; this corresponds to the choice of supergroup $\gamma=\left(\Gamma, 0, \Gamma \times \mathbb{Z}_{2}\right)$ in [St2]. We recall that $R_{n}^{\text {spin }}(B \Gamma)$ is the bordism group of quadruples $(N, s, f, h)$, where $N$ is a spin manifold of dimension $n$ possibly with boundary, $s$ is a spin structure, $f: N \rightarrow B \Gamma$ is a continuous map, $h$ is a PSC metric on $\partial N$. Two such quadruples $(N, s, f, h),\left(N^{\prime}, s^{\prime}, f^{\prime}, h^{\prime}\right)$ are said to be bordant if: (i) there is a bordism $(V, S, F, H)$ between $\left(\partial N,\left.s\right|_{\partial N},\left.f\right|_{\partial N}, h\right)$ and $\left(\partial N^{\prime},\left.s^{\prime}\right|_{\partial N^{\prime}},\left.f^{\prime}\right|_{\partial N^{\prime}}, h^{\prime}\right)$ viewed as elements in $\operatorname{Pos}_{n-1}^{\mathrm{spin}}(B \Gamma)$; (ii) the closed (glued) spin manifold $N \cup_{\partial N} V \cup_{\partial N^{\prime}} N^{\prime}$ is the boundary of some spin manifold $W$. 
Moreover, there is a spin structure $S_{W}$ on $W$ and a continuous map $E_{W}: W \rightarrow B \Gamma$ restricting to $s$ (resp. $s^{\prime}, S$ ) and $f$ (resp. $f^{\prime}$, resp. $F$ ) on $N$ (resp. $N^{\prime}$, resp. $V$ ).

We also recall that there exists a long exact sequence

$$
\cdots \rightarrow \Omega_{n+1}^{\mathrm{spin}}(B \Gamma) \rightarrow R_{n+1}^{\mathrm{spin}}(B \Gamma) \stackrel{\partial}{\longrightarrow} \operatorname{Pos}_{n}^{\mathrm{spin}}(B \Gamma) \rightarrow \Omega_{n}^{\mathrm{spin}}(B \Gamma) \rightarrow \cdots
$$

\section{A Product-Formula for the Higher Eta-Invariant}

Let $N^{n}$ be a spin manifold with a fixed spin structure $s$ and let $f: N \rightarrow B \Gamma$ be a continuous map. We assume that $N$ admits a metric $g$ of PSC. We can consider the Dirac operator $\not D$ associated to $s$ and $g$. We also have the $\Gamma$-covering $f^{*} E \Gamma$ with the $\Gamma$-invariant Riemannian metric $\widetilde{g}$ induced by $g$. We denote by $\widetilde{D}$ the lifted Dirac operator and by $\mathcal{D}$ the Dirac operator on $N$ twisted by the flat bundle

$$
\mathcal{V}_{f}=f^{*} E \Gamma \times_{\Gamma} \mathcal{B}_{\Gamma}^{\infty},
$$

here $\mathcal{B}_{\Gamma}^{\infty}$ denotes the Connes-Moscovici algebra [CM]. As $g$ has PSC, the Dirac operator $\mathcal{D}$ is invertible in the $\mathcal{B}_{\Gamma}^{\infty}$-Mishchenko-Fomenko calculus. Thus, according to [L2, §4.4], [L3] the higher eta-invariant $\tilde{\eta}$ of $\mathcal{D}$ is well defined (see also Section 2 of [LP1] and [LP3, Appendix]); it is an element in the vector space of noncommutative differential forms modulo graded commutators: $\bar{\Omega}_{*}\left(\mathcal{B}_{\Gamma}^{\infty}\right)=$ $\Omega_{*}\left(\mathcal{B}_{\Gamma}^{\infty}\right) / \overline{\left[\Omega_{*}\left(\mathcal{B}_{\Gamma}^{\infty}\right), \Omega_{*}\left(\mathcal{B}_{\Gamma}^{\infty}\right)\right]}$. By definition $\tilde{\eta}=\int_{0}^{+\infty} \tilde{\eta}(t) \mathrm{d} t$ with

$$
\widetilde{\eta}(t)= \begin{cases}\frac{2}{\sqrt{\pi}} \operatorname{STR}_{\mathrm{Cl}(1)} \mathcal{Y} \mathcal{D} \exp -\left(t \mathcal{Y D}+\nabla \otimes \mathrm{Id}_{\mathbb{C}^{2}}\right)^{2}, & \text { if } n \text { is odd, } \\ \frac{2}{\sqrt{\pi}} \operatorname{STR} \mathcal{D} \exp -(t \mathcal{D}+\nabla)^{2}, & \text { if } n \text { is even, }\end{cases}
$$

where $\mathcal{Y}=\left(\begin{array}{ll}0 & 1 \\ 1 & 0\end{array}\right)$ and $\nabla$ is the $\mathcal{B}_{\Gamma}^{\infty}$-connection defined by Lott in [L1] p. 436.

Recall that $\Omega_{*}\left(\mathcal{B}_{\Gamma}^{\infty}\right)$ is an algebra; whereas $\bar{\Omega}_{*}\left(\mathcal{B}_{\Gamma}^{\infty}\right)$ is only a vector space [Ka, L1]. We shall denote the higher eta-invariant associated to this Dirac operator by $\widetilde{\eta}(N, s, f, g)$; as in the case of the usual eta-invariant, $\widetilde{\eta}(N, s, f, g)$ depends in a nontrivial way on the choice of $g, f$, and $s$. This follows, for example, by the variational formulae proved in [L1]; in particular $\widetilde{\eta}(N, s, f, g)$ does not descend to $\operatorname{Pos}_{n}^{\text {spin }}(B \Gamma)$.

Let $(N, s, f, g)$ be a quadruple as above and let $(M, \sigma, \phi)$ be a triple as in the definition of $\Omega_{n}^{\text {spin }}(B \Delta)$. Let $h$ be a smooth metric on $M$; then for $\epsilon>0$ small enough, $(1 / \epsilon h \times g)$ is a metric of PSC on $M \times N$. Let us also consider the product spin structure and the product classifying map $\phi \times f$ into $B(\Delta \times \Gamma)$. We consider the following subalgebra of the reduced $C^{*}$-algebra $C_{r}^{*}(\Delta \times \Gamma): \mathcal{A}_{\Delta \times \Gamma}=\mathcal{B}_{\Delta}^{\infty} \widehat{\otimes} \mathcal{B}_{\Gamma}^{\infty}$.

The classifying map $\phi \times f$ defines a $\Delta \times \Gamma$-covering $\widetilde{M \times N}$ of $M \times N$. We then consider the flat $\mathcal{A}_{\Delta \times \Gamma}$-vector bundle over $M \times N$ defined as the fiber product 
$\mathcal{V}_{\phi \widehat{\otimes} f}=(\widetilde{M \times N}) \times_{(\Delta \times \Gamma)} \mathcal{A}_{\Delta \times \Gamma}$. These data define a $\mathcal{A}_{\Delta \times \Gamma}$-linear Dirac operator $\mathcal{D}_{M \times N}$ acting on $C^{\infty}\left(M \times N ; \mathcal{V}_{\phi \widehat{\otimes} f} \otimes S_{M \times N}\right)$. We shall also consider the connection $\nabla_{M \times N}$ introduced by Lott (see [L1, p. 456]) acting on

$$
C^{\infty}\left(M \times N ; \Omega_{*}\left(\mathcal{A}_{\Delta \times \Gamma}\right) \otimes_{\mathcal{A}_{\Delta \times \Gamma}} \mathcal{V}_{\phi \widehat{\otimes} f} \otimes S_{M \times N}\right)
$$

In the sequel we shall use the natural map (see [Co, p. 104]) $\pi: \Omega_{*}\left(\mathcal{A}_{\Delta \times \Gamma}\right) \rightarrow$ $\Omega_{*}\left(\mathcal{B}_{\Delta}^{\infty}\right) \widehat{\otimes} \Omega_{*}\left(\mathcal{B}_{\Gamma}^{\infty}\right)$; we shall still denote by $\pi$ the induced map

$$
\begin{aligned}
& C^{\infty}\left(M \times N ; \Omega_{*}\left(\mathcal{A}_{\Delta \times \Gamma}\right) \otimes_{\mathcal{A}_{\Delta \times \Gamma}} \mathcal{V}_{\phi \widehat{\otimes} f} \otimes S_{M \times N}\right) \\
& \quad \rightarrow C^{\infty}\left(M \times N ; \Omega_{*}\left(\mathcal{B}_{\Delta}^{\infty}\right) \widehat{\otimes} \Omega_{*}\left(\mathcal{B}_{\Gamma}^{\infty}\right) \otimes_{\mathcal{A}_{\Delta \times \Gamma}} \mathcal{V}_{\phi \widehat{\otimes} f} \otimes S_{M \times N}\right) .
\end{aligned}
$$

Using $\mathcal{D}_{M \times N}$ and $\nabla_{M \times N}$ one can define a higher eta-invariant, denoted $\widetilde{\eta}(M \times N) \epsilon$ $\bar{\Omega}_{*}\left(\mathcal{A}_{\Delta \times \Gamma}\right)$. The next Lemma will provide a product-formula for $\widetilde{\eta}(M \times N)$; it extends results by Atiyah-Patodi-Singer [APS3, p. 84] and Lott [L2, p. 205].

PROPOSITION 2.1. For the higher eta-invariant $\widetilde{\eta}(M \times N)$, the following formula holds modulo graded commutators and $\mathrm{d} \Omega_{*}\left(\mathcal{B}_{\Delta}^{\infty}\right) \widehat{\otimes} \Omega_{*}\left(\mathcal{B}_{\Gamma}^{\infty}\right)+\Omega_{*}\left(\mathcal{B}_{\Delta}^{\infty}\right) \widehat{\otimes}$ $\mathrm{d} \Omega_{*}\left(\mathcal{B}_{\Gamma}^{\infty}\right)$ :

$$
\pi(\widetilde{\eta}(M \times N))=C(m, n) \int_{M} \widehat{A}(M) \wedge \omega_{\phi^{*}(E \Delta)} \widehat{\otimes} \widetilde{\eta}(N, s, f, g) .
$$

In this formula $\omega_{\phi^{*}(E \Delta)}$ is the closed biform $\in \Omega^{*}(M) \widehat{\otimes} \bar{\Omega}_{*}\left(\mathcal{B}_{\Delta}^{\infty}\right)$ introduced by Lott ([L1] p. 436), and $C(m, n)$ is a nonzero constant depending only on $m$ and $n$.

Proof. We will treat only the case, where $m$ and $n$ are both odd. Recall that $S_{M}, S_{N}$ denote the bundle of spinors over $M$ and $N$, respectively. Then the spinor bundle $S_{M \times N}$ over $M \times N$ can be identified with $S_{M} \otimes S_{N} \otimes \mathbb{C}^{2}$. Let $\mathcal{Y}$ and $\mathcal{I}$ be the two anticommuting involutions of $\mathbb{C}^{2}$ defined by

$$
\mathcal{Y}=\left(\begin{array}{ll}
0 & 1 \\
1 & 0
\end{array}\right), \quad \mathcal{I}=\left(\begin{array}{cc}
0 & \sqrt{-1} \\
-\sqrt{-1} & 0
\end{array}\right)
$$

Then for the product metric on $M \times N$ and any $\alpha \in T_{x}^{*} M, \beta \in T_{y}^{*} N$, the clifford action on $S_{M} \otimes S_{N} \otimes \mathbb{C}^{2}$ is given by: $\operatorname{cl}(\alpha)=\operatorname{cl}_{M}(\alpha) \otimes \operatorname{Id} \otimes \mathcal{Y}, \operatorname{cl}(\beta)=\operatorname{Id} \otimes \operatorname{cl}_{N}(\beta) \otimes$ $\mathcal{I}$. The (generalized) Dirac operator $\mathcal{D}_{M \times N}$ introduced above acts on the sections of the $\mathcal{A}_{\Delta \times \Gamma^{-}}$-bundle $\mathcal{V}_{\phi \widehat{\otimes} f} \otimes S_{M} \otimes S_{N} \otimes \mathbb{C}^{2}$ over $M \times N$; we shall endow this bundle with the $\mathbb{Z}_{2}$-grading induced by the decomposition $\mathbb{C}^{2}=\mathbb{C} \oplus \mathbb{C}$. It's easy to see that the operator $\mathcal{D}_{M \times N}$ is equal to $\mathcal{D}_{M} \mathcal{Y}+\mathcal{D}_{N} \mathcal{I}$ (remark that $\mathcal{V}_{\phi \widehat{\otimes} f}=\mathcal{V}_{\phi} \widehat{\otimes} \mathcal{V}_{f}$ ). The higher eta-invariant $\widetilde{\eta}(M \times N))$ is by definition equal to $\int_{0}^{+\infty} \widetilde{\eta}(t) \mathrm{d} t$, where the local eta-integrand $\widetilde{\eta}(t)$ is defined to be

$$
\tilde{\eta}(t)=2 / \sqrt{\pi} \cdot \operatorname{STR} \mathcal{D}_{M \times N} \exp \left(-\left(t \mathcal{D}_{M \times N}+\nabla_{M \times N}\right)^{2}\right) .
$$

Next we introduce the connection $\nabla_{M \times N}^{\otimes}$ given by

$$
\nabla_{M \times N}^{\otimes}=\nabla_{M} \otimes \operatorname{Id}_{\mathbb{C}^{2}} \otimes \mathrm{Id}+\mathrm{Id} \otimes \operatorname{Id}_{\mathbb{C}^{2}} \otimes \nabla_{N}
$$


acting on

$$
C^{\infty}\left(M \times N ; \Omega_{*}\left(\mathcal{B}_{\Delta}^{\infty}\right) \widehat{\otimes} \Omega_{*}\left(\mathcal{B}_{\Gamma}^{\infty}\right) \otimes \mathcal{V}_{\phi} \widehat{\otimes} \mathcal{V}_{f} \otimes S_{M \times N}\right)
$$

We observe that $\pi \circ \nabla_{M \times N}=\nabla_{M \times N}^{\otimes}$; moreover Duhamel's formula implies that for each real $t>0$ one has

$$
\pi \circ \mathcal{D}_{M \times N} \exp -\left(t \mathcal{D}_{M \times N}+\nabla_{M \times N}\right)^{2}=\mathcal{D}_{M \times N} \exp -\left(t \mathcal{D}_{M \times N}+\nabla_{M \times N}^{\otimes}\right)^{2} .
$$

Thus the product formula (2.2) will follow from the following:

LEMMA 2.2. The following formula holds:

$$
\begin{array}{r}
\int_{0}^{+\infty} \frac{2}{\sqrt{\pi}} \operatorname{STR} \mathcal{D}_{M \times N} \exp -\left(t \mathcal{D}_{M \times N}+\nabla_{M \times N}^{\otimes}\right)^{2} \mathrm{~d} t \\
=C(m, n) \int_{M} \widehat{A}(M) \wedge \omega_{\phi^{*}(E \Delta)} \widehat{\otimes} \tilde{\eta}(N, s, f, g),
\end{array}
$$

modulo graded commutators and $\mathrm{d} \Omega_{*}\left(\mathcal{B}_{\Delta}^{\infty}\right) \widehat{\otimes} \Omega_{*}\left(\mathcal{B}_{\Gamma}^{\infty}\right)+\Omega_{*}\left(\mathcal{B}_{\Delta}^{\infty}\right) \widehat{\otimes} \mathrm{d} \Omega_{*}\left(\mathcal{B}_{\Gamma}^{\infty}\right)$.

Proof. Using supersign conventions, one checks easily that

$$
\left(t \mathcal{D}_{M \times N}+\nabla_{M \times N}^{\otimes}\right)^{2}=\left(t \mathcal{D}_{M} \mathcal{Y}+\nabla_{M} \operatorname{Id}_{\mathbb{C}^{2}}\right)^{2}+\left(t \mathcal{D}_{N} \mathcal{I}+\nabla_{N} \operatorname{Id}_{\mathbb{C}^{2}}\right)^{2} .
$$

Duhamel's expansion reads explicitly

$$
\begin{aligned}
\exp - & \left(t \mathcal{D}_{M} \mathcal{Y}+\nabla_{M} \operatorname{Id}_{\mathbb{C}^{2}}\right)^{2} \\
= & \exp \left(-t^{2} \mathcal{D}_{M}^{2}\right) \operatorname{Id}_{\mathbb{C}^{2}}+\sum_{k=1}^{+\infty}(-1)^{k} \int_{0}^{1} \int_{0}^{1-u_{1}} \ldots \int_{0}^{1-u_{1}-\ldots-u_{k-1}} \\
& \exp \left(-u_{1} t^{2} \mathcal{D}_{M}^{2}\right) \mathcal{X}_{t} \ldots \exp \left(-\left(1-u_{1}-\ldots-u_{k-1}\right) t^{2} \mathcal{D}_{M}^{2}\right) \mathrm{d} u_{k} \ldots \mathrm{d} u_{1},
\end{aligned}
$$

where we have set

$$
\mathcal{X}_{t}=\nabla_{M}^{2} \operatorname{Id}_{\mathbb{C}^{2}}+t\left(\mathcal{D}_{M} \nabla_{M}+\nabla_{M} \mathcal{D}_{M}\right) \mathcal{Y} .
$$

Moreover, we recall from [L2, p. 215] that $\mathcal{D}_{M} \nabla_{M}+\nabla_{M} \mathcal{D}_{M}$ and $\nabla_{M}^{2}$ are operators that increase the degree of differential forms on $\mathcal{B}_{\Delta}^{\infty}$ by one and two, respectively. Therefore, using the previous Duhamel's expansion and the fact that $\mathcal{Y}^{2}=\operatorname{Id}_{\mathbb{C}^{2}}$, one sees easily that for any real $t>0$

$$
\exp -\left(t \mathcal{D}_{M} \mathcal{Y}+\nabla_{M} \operatorname{Id}_{\mathbb{C}^{2}}\right)^{2}=\operatorname{Even}(t, M) \operatorname{Id}_{\mathbb{C}^{2}}+\operatorname{Odd}(t, M) \mathcal{Y},
$$

where Even $(t, M)(\operatorname{resp}$. Odd $(t, M))$ is defined as the sum of a series of operators acting on the set of sections of the bundle $\Omega_{*}\left(\mathcal{B}_{\Delta}^{\infty}\right) \widehat{\otimes}_{\mathcal{B}_{\Delta}^{\infty}} \mathcal{V}_{\phi} \otimes S_{M}$ which increase the degree of differential form on $\mathcal{B}_{\Delta}^{\infty}$ by an even (resp. odd) number. Of course, Even $(t, M)$ and $\operatorname{Odd}(t, M)$ are identified with diagonal operators when they act on the sections of the tensor product of the previous bundle with $\mathbb{C}^{2}$. Similarly, one can write for any real $t>0$

$$
\exp -\left(t \mathcal{D}_{N} \mathcal{I}+\nabla_{N} \operatorname{Id}_{\mathbb{C}^{2}}\right)^{2}=\operatorname{Even}(t, N) \operatorname{Id}_{\mathbb{C}^{2}}+\operatorname{Odd}(t, N) \mathcal{I} \text {. }
$$


For the proof of the Lemma we shall need the following intermediate result.

SUBLEMMA 2.3. For any real $t>0$ one has

$$
\begin{aligned}
\operatorname{STR} & \mathcal{D}_{M} \mathcal{Y} \exp \left(-\left(t \mathcal{D}_{M \times N}+\nabla_{M \times N}^{\otimes}\right)^{2}\right) \\
= & \frac{2}{\sqrt{-1}} \mathrm{TR}_{\text {even }} \mathcal{D}_{M} \mathrm{e}^{-\left(t \mathcal{D}_{M}+\nabla_{M}\right)^{2}} \widehat{\otimes} \mathrm{TR}_{\text {odd }} \mathrm{e}^{-\left(t \mathcal{D}_{N}+\nabla_{N}\right)^{2}},
\end{aligned}
$$

where the subscript even (odd) means that one projects onto the space of differential forms (on $\mathcal{B}_{\Delta}^{\infty}$ or $\mathcal{B}_{\Gamma}^{\infty}$ ) of even (odd) degrees.

Proof. Using the three formulas, (2.3), (2.4), (2.5), one sees that for any real $t>0$

$$
\begin{array}{rl}
\mathcal{D}_{M} & \mathcal{Y} \exp \left(-\left(t \mathcal{D}_{M \times N}+\nabla_{M \times N}^{\otimes}\right)^{2}\right) \\
= & \left(\mathcal{D}_{M} \operatorname{Even}(t, M) \mathcal{Y}+\mathcal{D}_{M} \operatorname{Odd}(t, M) \operatorname{Id}_{\mathbb{C}^{2}}\right) \circ\left(\operatorname{Even}(t, N) \operatorname{Id}_{\mathbb{C}^{2}}+\right. \\
\quad & +\operatorname{Odd}(t, N) \mathcal{I}) .
\end{array}
$$

As the supertraces of the matrixes $\mathcal{Y}, \mathcal{I}, \operatorname{Id}_{\mathbb{C}^{2}}$ vanish and the supertrace of $\mathcal{Y} \circ \mathcal{I}$ is equal to $-2 \sqrt{-1}$, one then gets

$$
\begin{aligned}
& \operatorname{STR} \mathcal{D}_{M} \mathcal{Y} \exp \left(-\left(t \mathcal{D}_{M \times N}+\nabla_{M \times N}^{\otimes}\right)^{2}\right) \\
& \quad=-2 \sqrt{-1} \operatorname{TR}\left(\mathcal{D}_{M} \operatorname{Even}(t, M)\right) \widehat{\otimes} \operatorname{TR} \operatorname{Odd}(t, N) .
\end{aligned}
$$

But analyzing Duhamel's expansion for $\mathrm{e}^{-\left(t \mathcal{D}_{M}+\nabla_{M}\right)^{2}}$ and $\mathrm{e}^{-\left(t \mathcal{D}_{N}+\nabla_{N}\right)^{2}}$, one recognizes that

$$
\begin{aligned}
& \operatorname{TR}\left(\mathcal{D}_{M} \text { Even }(t, M)\right)=\mathrm{TR}_{\text {even }} \mathcal{D}_{M} \mathrm{e}^{-\left(t \mathcal{D}_{M}+\nabla_{M}\right)^{2}}, \\
& \operatorname{TR} \operatorname{Odd}(t, N)=\mathrm{TR}_{\text {odd }} \mathrm{e}^{-\left(t \mathcal{D}_{N}+\nabla_{N}\right)^{2}},
\end{aligned}
$$

which proves Sublemma 2.3.

Now we go back to the proof of Lemma 2.2 (and thus of Proposition 2.1), as $\mathcal{D}_{N}$ is invertible, we see that

$$
\mathrm{TR}_{\text {odd }} \mathrm{e}^{-\left(t \mathcal{D}_{N}+\nabla_{N}\right)^{2}}
$$

tends to 0 as $t \rightarrow+\infty$; as (2.6) does not depend on $t$, modulo exact forms, Sublemma 2.3 shows that for each real $t>0 \mathrm{STR} \mathcal{D}_{M} \mathcal{Y} \exp \left(-\left(t \mathcal{D}_{M \times N}+\nabla_{M \times N}^{\otimes}\right)^{2}\right)$ is an element of $\Omega_{*}\left(\mathcal{B}_{\Delta}^{\infty}\right) \widehat{\otimes} \mathrm{d} \Omega_{*}\left(\mathcal{B}_{\Gamma}^{\infty}\right)$. Next, similarly, one proves that

$$
\begin{aligned}
& \operatorname{STR} \mathcal{D}_{N} \mathcal{I} \exp \left(-\left(t \mathcal{D}_{M \times N}+\nabla_{M \times N}^{\otimes}\right)^{2}\right) \\
& \quad=2 \sqrt{-1} \mathrm{TR}_{\text {odd }} \mathrm{e}^{-\left(t \mathcal{D}_{M}+\nabla_{M}\right)^{2}} \widehat{\otimes} \mathrm{TR}_{\text {even }} \mathcal{D}_{N} \mathrm{e}^{-\left(t \mathcal{D}_{N}+\nabla_{N}\right)^{2}} .
\end{aligned}
$$

Recall that

$$
\widetilde{\eta}(N, s, f, g)(t)=\frac{2}{\sqrt{\pi}} \operatorname{STR}_{\mathrm{Cl}(1)}\left(\mathcal{Y} \mathcal{D}_{N} \exp -\left(t \mathcal{Y} \mathcal{D}_{N}+\nabla_{N} \otimes \operatorname{Id}_{\mathbb{C}^{2}}\right)^{2}\right) .
$$


Proceeding as in the proof of Sublemma 2.3, one checks easily that

$$
\widetilde{\eta}(N, s, f, g)(t)=\frac{2}{\sqrt{\pi}} \mathrm{TR}_{\mathrm{even}} \mathcal{D}_{N} \mathrm{e}^{-\left(t \mathcal{D}_{N}+\nabla_{N}\right)^{2}} .
$$

Moreover the index theorem of Lott ([L1] p. 440 and also [L2] p. 219) shows that $\mathrm{TR}_{\text {odd }} \mathrm{e}^{-\left(t \mathcal{D}_{M}+\nabla_{M}\right)^{2}}$ is precisely equal to $\int_{M} \widehat{A}(M) \wedge \omega_{f_{1}}{ }^{*}(E \Delta)$ modulo an exact form which depends on $t$ and is integrable as $t \rightarrow 0^{+}$. One then obtains immediately Lemma 2.2 and thus Proposition 2.1.

\section{Spin Bordism Groups and Higher APS Index Theory}

We now consider an element $\left[M, s, f, g_{\partial}\right] \in R_{n}^{\text {spin }}(B \Gamma)$. Let ${ }^{b} g$ be a $b$-Riemannian metric [Me] on $M$ extending $g_{\partial}$. To such a quadruple $\left(M, s, f, g_{\partial}\right)$ we can associate the $b$-index class [LP1, p. 80, 88] $\operatorname{Ind}_{b}(\mathcal{D}) \in K_{*}\left(\mathcal{B}_{\Gamma}^{\infty}\right) \equiv K_{*}\left(C_{r}^{*}(\Gamma)\right)$ of the Dirac operator defined by $s$ and ${ }^{b} g$ twisted by the flat bundle $\mathcal{V}_{f}=f^{*} E \Gamma \times_{\Gamma} \mathcal{B}_{\Gamma}^{\infty}$. This index class is well defined since, by definition of $R_{n}^{\text {spin }}(B \Gamma), g_{\partial}$ is of PSC; thus the boundary operator is invertible in the Mishchenko-Fomenko calculus and the operator is $C_{r}^{*}(\Gamma)$-Fredholm according to the results in [LP1, p. 80]. Alternatively, let $g$ be a Riemannian metric on $M$ extending $g_{\partial}$. Then, we could define a AtiyahPatodi-Singer index class, as in [Wu2]; it can be proved that the two index classes so defined are equal, see [LP4, Theorem 5]. The following is a complex analogue of results of Bunke [Bu] and Stolz [St2].

PROPOSITION 3.1. The exists a well-defined morphism of groups ${ }^{b} \Theta_{\mathbb{C}}: R_{n}^{\mathrm{spin}}$ $(B \Gamma) \rightarrow K_{*}\left(C_{r}^{*}(\Gamma)\right)$; it associates to a class $\left[M, s, f, g_{\partial}\right]$ the $b$-index of the $C_{r}^{*}(\Gamma)$ Dirac operator with coefficients in $\mathcal{V}_{f}$ defined by $s$ and an extension of $g_{\partial}$ to $M$.

Proof. We have to show that the map is well defined. Obviously the index class does not depend on the choice of the extending $b$-metric as the Dirac operators associated to two different extensions are $C_{r}^{*}(\Gamma)$-homotopic. Next we have to show that the class is the same for two bordant quadruples $\left(M, s, f, g_{\partial M}\right)$, $\left(M^{\prime}, s^{\prime}, f^{\prime}, g_{\partial M^{\prime}}\right)$. This follows from a splitting formula for the $b$-index together with the cobordism invariance of the index. The splitting formula for APS-index classes, and thus $b$-index classes, is proved in detail in [LP4, Theorem 8] following ideas in [DZ1, p. 314]. The Proposition is proved.

Remark 1. We have defined a morphism of groups ${ }^{b} \Theta_{\mathbb{C}}: R_{n}^{\text {spin }}(B \Gamma) \rightarrow K_{*}$ $\left(C_{r}^{*}(\Gamma)\right)$; it should be interesting to compare this morphism with the composition of the morphism $\Theta: R_{n}^{\text {spin }}(B \Gamma) \rightarrow K O_{*}\left(C_{r}^{*}(\Gamma)_{\mathbb{R}}\right)$ defined by Bunke and Stolz (see [Bu, St2]) with the natural map $K O_{*}\left(C_{r}^{*}(\Gamma)_{\mathbb{R}}\right) \rightarrow K_{*}\left(C_{r}^{*}(\Gamma)\right)$. See [L-M, p. 143] in the case of the trivial group.

Remark 2. In this paper we shall concentrate on the information we can get by taking the composition of ${ }^{b} \Theta_{\mathbb{C}}: R_{n}^{\text {spin }}(B \Gamma) \rightarrow K_{*}\left(C_{r}^{*}(\Gamma)\right)=K_{*}\left(\mathcal{B}_{\Gamma}^{\infty}\right)$ with a 
noncommutative Chern character (see below) and by then pairing the result with suitable cyclic cocycles. In other words, we want to consider numerical higher invariants associated to bordism classes; on the one hand this amounts to a loss of information, on the other hand these numerical invariants are sometimes easier to deal with than the $K$-theoretic counterparts. Notice that there is no real analogue of the Chern character map, not even in the case $\Gamma=\{1\}$ [ASV, LM].

Let $\bar{H}_{*}\left(\mathcal{B}_{\Gamma}^{\infty}\right)$ be the (topological) noncommutative de Rham homology defined by the complex of noncommutative differential forms on $\mathcal{B}_{\Gamma}^{\infty}$ [see Ka, §4.1]. Thanks to the work of Karoubi [Ka] we can take the Chern character $K_{0}\left(\mathcal{B}_{\Gamma}^{\infty}\right) \rightarrow$ $\bar{H}_{\text {even }}\left(\mathcal{B}_{\Gamma}^{\infty}\right)$. In particular the Chern character of the $b$-index class associated to a quadruple $\left(M, s, f, g_{\partial M}\right)$ is well defined when $M$ has even dimension. In order to deal with the odd dimensional case, we use suspension as in [MP, LLP]. Let $\tau^{\prime}$ be the generator for $H^{1}(\mathbb{Z} ; \mathbb{Z}) \subset H^{1}(\mathbb{Z} ; \mathbb{C})$. Put $\tau=\sqrt{-1} \tau^{\prime} / 2 \pi \in H^{1}(\mathbb{Z} ; \mathbb{C})$. There is a natural desuspension map $\langle\cdot, \tau\rangle: \bar{H}_{*}\left(\mathcal{B}_{\Gamma \times \mathbb{Z}}^{\infty}\right) \rightarrow \bar{H}_{*}\left(\mathcal{B}_{\Gamma}^{\infty}\right)$.

DEFINITION 3.2. We define a morphism of groups ${ }^{b} \alpha_{n}: R_{n}^{\mathrm{spin}}(B \Gamma) \rightarrow \bar{H}_{*}\left(\mathcal{B}_{\Gamma}^{\infty}\right)$ as follows. If $n=2 m$ then ${ }^{b} \alpha_{2 m}:=\mathrm{Ch}$ o $^{b} \Theta_{\mathbb{C}}$. If $n=2 m-1$ then ${ }^{b} \alpha_{2 m-1}[M, s, f$, $\left.g_{\partial M}\right]=\left\langle{ }^{b} \alpha_{2 m}\left[M \times S^{1}, f \times f_{S^{1}}, s \times s_{S^{1}}, g_{\partial M} \times g_{S^{1}}\right], \tau\right\rangle$. If $n$ is even (resp. odd) the map ${ }^{b} \alpha$ has values in $\bar{H}_{\text {even }}\left(\mathcal{B}_{\Gamma}^{\infty}\right)\left(\operatorname{resp} . \bar{H}_{\text {odd }}\left(\mathcal{B}_{\Gamma}^{\infty}\right)\right)$.

Using the higher APS index theorem in [LP1] (§14) and Proposition 2.1 (in this case $\mathcal{B}_{\Gamma \times \mathbb{Z}}^{\infty} \simeq \mathcal{B}_{\Gamma}^{\infty} \widehat{\otimes} \mathcal{B}_{\mathbb{Z}}^{\infty}$ ), we immediatly obtain the following formula

$$
\begin{aligned}
{ }^{b} \alpha\left[M, s, f, g_{\partial}\right]= & {\left[\int_{M} \widehat{A}(M) \wedge \omega_{f^{*}(E \Gamma)}-\frac{1}{2} \widetilde{\eta}\left(\partial M, \partial s,\left.f\right|_{\partial M}, g_{\partial M}\right)\right] } \\
& \in \bar{H}_{*}\left(\mathcal{B}_{\Gamma}^{\infty}\right),
\end{aligned}
$$

with $\omega_{f^{*}(E \Gamma)}$ the bi-form introduced by Lott in [L1, p. 436].

\section{Distinguishing PSC Metrics through Higher Rho-Invariants}

We first define higher $L^{2}$ bordism invariants. We first deal with the case where $\Gamma$ is virtually nilpotent. We follow closely [L2, §4.6]. Under this additional assumption, the complex $\bar{\Omega}_{*}\left(\mathcal{B}_{\Gamma}^{\infty}\right)$ splits as the direct sum of sub-complexes labeled by the conjugacy classes $\langle\Gamma\rangle$ of $\Gamma$. Thus, $\widetilde{\eta}(N, s, f, g)$ splits as a direct sum

$$
\widetilde{\eta}(N, s, f, g)=\bigoplus_{\langle x\rangle \in\langle\Gamma\rangle} \tilde{\eta}(\langle x\rangle)
$$

The higher $\rho$-invariant associated to the quadruple $(N, s, f, g)$ is, by definition,

$$
\widetilde{\rho}(N, s, f, g)):=\bigoplus_{\langle x\rangle \neq\langle e\rangle} \tilde{\eta}(\langle x\rangle) .
$$

As pointed out in [L2, p. 222], the higher $\rho$-invariant has in fact values in $\bar{H}_{*}\left(\mathcal{B}_{\Gamma}^{\infty}\right)$. Notice that $\bar{H}_{*}\left(\mathcal{B}_{\Gamma}^{\infty}\right)$ splits as the direct sum $\bar{H}_{*}\left(\mathcal{B}_{\Gamma}^{\infty}\right)=\bar{H}_{*}^{\mathrm{t}}\left(\mathcal{B}_{\Gamma}^{\infty}\right) \oplus$ 
$\bar{H}_{*}^{\text {nt }}\left(\mathcal{B}_{\Gamma}^{\infty}\right)$ with the first group on the right-hand side associated to the subcomplex of $\bar{\Omega}_{*}\left(\mathcal{B}_{\Gamma}^{\infty}\right)$ labeled by the trivial conjugacy class and the second group associated to the subcomplexes labeled by the nontrivial conjugacy classes. Then $\widetilde{\rho}(N, s, f, g)$ has values in $\bar{H}_{*}^{\mathrm{nt}}\left(\mathcal{B}_{\Gamma}^{\infty}\right)$, and more precisely, in $\bar{H}_{\text {even }}^{\mathrm{nt}}\left(\mathcal{B}_{\Gamma}^{\infty}\right)$ if $N$ has odd dimension and $\bar{H}_{\text {odd }}^{\text {nt }}\left(\mathcal{B}_{\Gamma}^{\infty}\right)$ if $N$ has even dimension. For future reference we let $\pi^{\mathrm{t}}$ and $\pi^{\mathrm{nt}}$ be the natural projection maps.

That the higher $\rho$-invariant could be used in questions of PSC was already observed in [L2]. First of all it is proved in [L2, p. 221] that the higher $\rho$-invariants is a deformation invariant with respect to the metric $g$. This means that the higher $\rho$-invariant is locally constant on the space of PSC metrics on the spin manifold $N$. Secondly, it was remarked in [L2] that if the higher eta-invariant was the boundary correction term in a to-be-proved higher Atiyah-Patodi-Singer index theorem, then the higher $\rho$ invariant would be an obstruction to extending a PSC metric from the boundary $\partial M$ of a compact spin manifold to the entire manifold $M$, so as to have a product metric near the boundary (see [L2, p. 228]). The higher Atiyah-PatodiSinger index formula conjectured in [L2] was proved in [LP1, p. 97] and a precise form of Lott's remark is now stated in Section 15 there. Fundamental in both these applications of the higher $\rho$-invariant is the fact that Lott's biform is concentrated in the trivial conjugacy class. We shall now use once again this property in order to prove the following.

PROPOSITION 4.1. Let $\Gamma$ be virtually nilpotent. The higher $\rho$-invariant defines a group homomorphism

$$
\tilde{\rho}: \operatorname{Pos}_{n}^{\mathrm{spin}}(B \Gamma) \rightarrow \bar{H}_{*}^{\mathrm{nt}}\left(\mathcal{B}_{\Gamma}^{\infty}\right) \subset \bar{H}_{*}\left(\mathcal{B}_{\Gamma}^{\infty}\right) .
$$

Moreover, the following diagram is commutative:

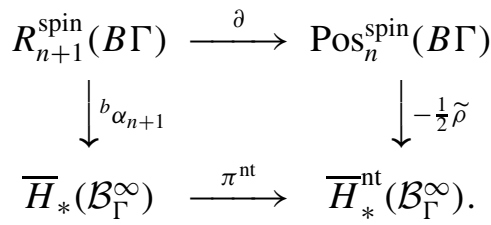

Proof. We need to prove that if $(N, s, f, g)$ is bordant to $\left(N^{\prime}, s^{\prime}, f^{\prime}, g^{\prime}\right)$ in $\operatorname{Pos}_{n}^{\text {spin }}(B \Gamma)$ then $\widetilde{\rho}(N, s, f, g)=\widetilde{\rho}\left(N^{\prime}, s^{\prime}, f^{\prime}, g^{\prime}\right)$. Let $(W, S, F, H)$ be a bordism between $(N, s, f, g)$ and $\left(N^{\prime}, s^{\prime}, f^{\prime}, g^{\prime}\right)$; then using the fact that $H$ is of PSC and the index formula of [LP1, p. 97][LP3], we obtain

$$
0=\int_{W} \widehat{A}\left(W, \nabla^{W}\right) \wedge \omega_{F^{*}(E \Gamma)}-\frac{1}{2} \widetilde{\eta}(N, s, f, g)+\frac{1}{2} \widetilde{\eta}\left(N^{\prime}, s^{\prime}, f^{\prime}, g^{\prime}\right),
$$

as the first summand on the right-hand side is concentrated on the trivial conjugacy class of $\Gamma$ (see [L1, p. 441]) we obtain immediately the equality $0=$ $\widetilde{\rho}(N, s, f, g)-\widetilde{\rho}\left(N^{\prime}, s^{\prime}, f^{\prime}, g^{\prime}\right)$. The commutativity of the diagram is an easy consequence of formula (3.1) and of the definition of $\widetilde{\rho}$. 
Remark 1. Let $\Xi=\left[\left(M, s, f, g_{\partial M}\right)\right] \in R_{n+1}^{\text {spin }}(B \Gamma)$, with $\Gamma$ virtually nilpotent. If $\widetilde{\rho}(\partial \Xi) \neq 0$ in $\bar{H}_{*}\left(\mathcal{B}_{\Gamma}^{\infty}\right)$ then $g_{\partial M}$ does not extend to a PSC metric on $M$. As already remarked (see [L2] and then [LP1, §15]) this follows from the definition of $\widetilde{\rho}$ and the higher APS index formula.

For an arbitrary finitely generated group the existence of the splitting (4.1) seems to be unknown (and possibly false). However, we can still consider interesting bordism invariants defined in terms of Lott's higher eta-invariants. So let $\Gamma$ be an arbitrary finitely generated group and let $\mathcal{B}_{\Gamma}^{\infty}$ be the Connes-Moscovici algebra. Consider the higher eta-invariant $\widetilde{\eta}(N, s, f, g)$. Recall (see [L2, p. 209]) that given $x \in \Gamma, x \neq e$, it is possible to define a cochain complex $C_{x}^{k}$ with $H_{x}^{k} \cong H^{k}\left(N_{\langle x\rangle}\right)$; here $N_{\langle x\rangle}$ is the quotient $Z_{x} \backslash\{x\}$, with $\{x\}$ equal to the cyclic group generated by $x$ and $Z_{x}$ the centralizer of $x$ in $\Gamma$. The cyclic cohomology of $\mathbb{C} \Gamma$ can be expressed in terms of $H^{k}\left(N_{\langle x\rangle}\right)$ ([Bur]). Given $\tau \in Z C_{x}^{k} \subset C_{x}^{k}$ there is a cyclic cocycle $\mathcal{S}_{\tau} \in Z C^{k}(\mathbb{C} \Gamma)$ defined as

$$
\mathcal{S}_{\tau}\left(\gamma_{0}, \ldots, \gamma_{k}\right)=\left\{\begin{array}{l}
0, \quad \text { if } \gamma_{0} \cdots \gamma_{k} \notin\langle x\rangle, \\
\tau\left(g, g \gamma_{0}, \ldots, g \gamma_{0} \ldots \gamma_{k-1}\right), \\
\text { if } \gamma_{0} \cdots \gamma_{k}=g^{-1} x g .
\end{array}\right.
$$

Let us assume that $\mathcal{S}_{\tau}$ extends to a cyclic cocycle of $\mathcal{B}_{\Gamma}^{\infty}$, we consider $\widetilde{\rho}\left(\mathcal{S}_{\tau}\right)$ $(N, s, f, g):=\left\langle\widetilde{\eta}(N, s, f, g) ; \mathcal{S}_{\tau}\right\rangle \in \mathbb{C}$ with $\langle\cdot ; \cdot\rangle$ the pairing between the noncommutative differential forms and the cyclic cocycles of $\mathcal{B}_{\Gamma}^{\infty}$. Proceeding as in Proposition 4.1 one proves the following.

PROPOSITION 4.2. The pairing $\left\langle\widetilde{\eta}(N, s, f, g) ; \mathcal{S}_{\tau}\right\rangle$ gives a well-defined map

$$
\widetilde{\rho}\left(\mathcal{S}_{\tau}\right): \operatorname{Pos}_{n}^{\text {spin }}(B \Gamma) \rightarrow \mathbb{C} .
$$

Remark 2. In the sequel we shall consider groups of the type $F \times \Delta$, with $F$ finite. Let $\langle x\rangle$ be a nontrivial conjugacy class of $F$ and consider the trace $\mathcal{S}_{\langle x\rangle}$ on $\mathbb{C} F$ defined by

$$
\mathcal{S}_{\langle x\rangle}\left(\sum_{\gamma \in F} a_{\gamma} \gamma\right)=\sum_{\gamma \in\langle x\rangle} a_{\gamma} .
$$

This 0-cocycle $\mathcal{S}_{\langle x\rangle}$ defines a pairing: $\bar{\Omega}_{*}\left(\mathcal{B}_{F \times \Delta}^{\infty}\right) \rightarrow \bar{\Omega}_{*}\left(\mathcal{B}_{\Delta}^{\infty}\right)$; proceeding as in Proposition 4.1 we see that the application $\widetilde{\rho}\left(\mathcal{S}_{\langle x\rangle}\right): \operatorname{Pos}_{n}^{\text {spin }}(B(F \times \Delta)) \rightarrow \bar{\Omega}_{*}\left(\mathcal{B}_{\Delta}^{\infty}\right)$ given by

$$
\operatorname{Pos}_{n}^{\mathrm{spin}}(B(F \times \Delta)) \ni[N, s, f, g] \rightarrow\left\langle\widetilde{\eta}(N, s, f, g) ; \mathcal{S}_{\langle x\rangle}\right\rangle \in \bar{\Omega}_{*}\left(\mathcal{B}_{\Delta}^{\infty}\right)
$$

is well defined.

Let us now concentrate on the problem of distinguishing nonbordant metrics of PSC. The following Proposition is directly inspired by the work of Botvinnik and Gilkey [Bo-Gi]. 
PROPOSITION 4.3. Let $N$ be a connected spin closed manifold of dimension $n \geqslant 5$ admitting one metric of PSC. We assume that there exists $\left[\left(N_{1}, s_{1}, f_{1}, g_{1}\right)\right] \in$ $\operatorname{Pos}_{n}^{\mathrm{spin}}\left(B \pi_{1}(N)\right)$ and a cyclic cocycle $\mathcal{S}_{\tau}$ as in Proposition 4.2 with the following properties: (1) $\left.\left\langle\widetilde{\eta}\left(N_{1}, s_{1}, f_{1}, g_{1}\right)\right) ; \mathcal{S}_{\tau}\right\rangle \neq 0$; (2) $\left[N_{1}, f_{1}, s_{1}\right]$ is a torsion element in $\Omega_{n}^{\text {spin }}\left(B \pi_{1}(N)\right)$. Then the space of PSC metrics on $N$ has an infinite number of pairwise nonbordant metrics.

Proof. By assumption there exists a $k \in \mathbb{N}^{*}$ such that $k\left[\left(N_{1}, s_{1}, f_{1}\right)\right]=0$ in $\Omega_{n}^{\text {spin }}\left(B \pi_{1}(N)\right)$. Let $f: N \rightarrow B \pi_{1}(N)$ be the classifying map of the universal cover; then for each $j \in \mathbb{N}, j \geqslant 2$

$$
[(N, s, f)]=[(N, s, f)]+j k\left[\left(N_{1}, s_{1}, f_{1}\right)\right] .
$$

By assumption there exists a metric of PSC on $N, g$. Thus $[(N, s, f, g)]+j k\left[\left(N_{1}\right.\right.$, $\left.\left.s_{1}, f_{1}, g_{1}\right)\right]$ is well defined in $\operatorname{Pos}_{n}^{\text {spin }}\left(B \pi_{1}(N)\right)$. By a well-known result of Miyazaki and Rosenberg (see e.g. Lemma 3.1 in [Bo-Gi]) we infer that $\forall j \in \mathbb{N} \backslash\{0,1\}$, there exists a metric $g_{j}$ of PSC such that

$$
\left[\left(N, s, f, g_{j}\right)\right]=[(N, s, f, g)]+j k\left[\left(N_{1}, s_{1}, f_{1}, g_{1}\right)\right] \text { in } \operatorname{Pos}_{n}^{\text {spin }}\left(B \pi_{1}(N)\right) .
$$

Here, it is crucial to work in the spin category, see [St1, p. 629]. Using the assumption

$$
\widetilde{\rho}\left(\mathcal{S}_{\tau}\right)\left[N_{1}, s_{1}, f_{1}, g_{1}\right]:=\left\langle\widetilde{\eta}\left(N_{1}, s_{1}, f_{1}, g_{1}\right) ; \mathcal{S}_{\tau}\right\rangle \neq 0
$$

and Proposition 4.2, we immediately obtain

$$
\widetilde{\rho}\left(\mathcal{S}_{\tau}\right)\left[N, s, f, g_{j}\right] \neq \widetilde{\rho}\left(\mathcal{S}_{\tau}\right)\left[N, s, f, g_{\ell}\right] \quad \text { if } \ell \neq j .
$$

This implies that $g_{j}$ and $g_{\ell}$, for $j \neq \ell$, are not bordant. The proof of the Proposition is complete.

Remark 3. In the case treated by Botvinnik and Gilkey, where $\pi(N)$ is finite, the torsion condition in the statement of the above Proposition is automatically satisfied. It is important to remark that the arguments given in [Bo-Gi] can be easily extended so as to treat infinite groups of a certain type. More precisely let $F$ be a finite group with $r_{2 n-1}(F)>0, n \geqslant 3$. Let $\Gamma=\Gamma^{\prime} \rtimes F$ with $\Gamma^{\prime}$ a finitely generated group.

CLAIM. If $N$ is a connected closed spin manifold of dimension $2 n-1$ admitting one metric of PSC and such that $\pi_{1}(N)=\Gamma$, then $N$ admits an infinite number of pairwise nonbordant metrics.

To prove the claim observe that, by assumption, there exists a split exact sequence $1 \rightarrow \Gamma^{\prime} \rightarrow \Gamma \rightarrow F \rightarrow 1$; by [Bo-Gi] there exist an element $\left[N_{1}, s_{1}, f_{1}, g_{1}\right] \in$ 
$\operatorname{Pos}_{2 n-1}^{\text {spin }}(B F)$ and a virtual representation $\phi \in R_{0}(F)$ such that the twisted etainvariant (i.e. the eta-invariant of the Dirac operator twisted by the flat bundle associated to $\phi$ ) is different from zero: $\eta\left[N_{1}, s_{1}, f_{1}, g_{1}\right](\phi) \neq 0$. As $\Omega_{2 n-1}^{\mathrm{spin}}(B F)$ is torsion there exists a $k \in \mathbb{N}^{*}$, such that $k\left[N_{1}, s_{1}, f_{1}\right]=0$. We let $i$ be the splitting map $i: F \rightarrow \Gamma$ and $p$ be the surjection $\Gamma \rightarrow F$ in the above exact sequence. We have $p \circ i=\mathrm{Id}$ by assumption and thus $B p \circ B i=\mathrm{Id}$. Notice that $k\left[N_{1}, s_{1}, B i \circ f_{1}\right]=0$. By applying $p_{*}$ to (4.7) above with $\left[\left(N_{1}, s_{1}, B i \circ f_{1}, g_{1}\right)\right]$ replacing $\left[\left(N_{1}, s_{1}, f_{1}, g_{1}\right)\right]$ and using $B p \circ B i=\mathrm{Id}$, we obtain a sequence of PSC metrics $\left\{g_{j}\right\}, j \geqslant 2$ such that:

$$
\begin{aligned}
& {\left[\left(N, s, B p \circ f, g_{j}\right)\right]} \\
& \quad=[(N, s, B p \circ f, g)]+j k\left[\left(N_{1}, s_{1}, f_{1}, g_{1}\right)\right] \quad \text { in } \operatorname{Pos}_{n}^{\text {spin }}(B F) .
\end{aligned}
$$

Thus

$$
\eta\left(N, s, B p \circ f, g_{j}\right)(\phi) \neq \eta\left(N, s, B p \circ f, g_{\ell}\right)(\phi) \quad \text { if } \ell \neq j .
$$

This implies that $g_{j}$ and $g_{\ell}$ are not bordant for if they were, then, by definition, $\left[N, s, f, g_{j}\right]=\left[N, s, f, g_{\ell}\right]$, which would imply, by functoriality, that $[(N, s, B p \circ$ $\left.\left.f, g_{j}\right)\right]=\left[\left(N, s, B p \circ f, g_{\ell}\right)\right]$ which, in turn, would contradict (4.8). This proves the claim.

One can also prove, by a similar reasoning, that for the moduli space $\mathcal{M}(N)=$ $\mathcal{R}^{+}(N) / \operatorname{Diff}(N)$ one has: $\left|\pi_{0}(\mathcal{M}(N))\right|=\infty$. As we shall not need this result, we omit the proof.

End of Remark 3.

Remark 4. Notice that the conclusion of Proposition 4.3 can be easily extended to the case $\pi_{1}(N)=F \times \Delta, F$ finite, if we replace the first assumption with the following one:

$\left(1^{\prime}\right)$ there exists a nontrivial conjugacy class $\langle x\rangle$ in $F$ such that $\widetilde{\rho}\left(S_{\langle x\rangle}\right)\left[N_{1}\right.$, $\left.s_{1}, f_{1}, g_{1}\right] \neq 0$ in $\bar{\Omega}\left(\mathcal{B}_{\Delta}^{\infty}\right)$.

\section{Proof of Theorem 0.1}

We are now in the position of proving the main result stated in the Introduction.

PROPOSITION 5.1. Let $N$ be a connected closed spin manifold of dimension $n$ such that $\pi_{1}(N)=\Gamma \times \Delta$ with $\Gamma=\Gamma^{\prime} \rtimes F$, where $F$ is finite and $r_{m}(F)>0$ for some odd integer $m, 5 \leqslant m<n$. We assume that $N$ admits one metric of PSC and that there exists $\mathcal{X}=[X, s, f] \in \Omega_{n-m}^{\mathrm{spin}}(B \Delta) / \Omega_{n-m}^{\mathrm{spin},+}(B \Delta)$ such that $\int_{X} \widehat{A}\left(X, \nabla^{X}\right) \wedge \omega_{f^{*}(E \Delta)} \neq 0$ in $\bar{\Omega}_{*}\left(\mathcal{B}_{\Delta}^{\infty}\right)$. Then there exists an infinite number of pairwise nonbordant PSC metrics on $N$.

Proof. We will prove the Proposition when $\Gamma=F$ and leave the extension to the semidirect product case to the reader (the steps are the same as in the proof of the claim in Remark 3 of the previous Section). Using again the results of 
[Bo-Gi] we know that there exists a bordism class $\left[L, s_{L}, f_{L}, g_{L}\right] \in \operatorname{Pos}_{m}(B F)$ and a virtual representation $\phi \in R_{0}(F)$ such that

$$
\eta\left[L, s_{L}, f_{L}, g_{L}\right](\phi) \neq 0 .
$$

We also know that $B F \times B \Delta=B(F \times \Delta) \equiv B\left(\pi_{1}(N)\right)$. Let $X$ and $f: X \rightarrow B \Delta$ be as in the hypothesis; let $s_{X}$ be a spin structure on $X$ and $g_{X}$ a Riemannian metric. We consider $\left[L \times X, s_{L} \times s_{X}, f_{L} \times f, g_{L} \times \frac{1}{\epsilon} g_{X}\right] \in \operatorname{Pos}_{n}\left(B \pi_{1}(N)\right)$ with $\epsilon>0$ small enough so that $g_{L} \times \frac{1}{\epsilon} g_{X}$ have PSC. Then Proposition 2.1 shows that

$$
\begin{aligned}
& \tilde{\eta}\left[L \times X, s_{L} \times s_{X}, f_{L} \times f, g_{L} \times \frac{1}{\epsilon} g_{X}\right](\phi) \\
& \quad=C \cdot \eta\left(L, s_{L}, f_{L}, g_{L}\right)(\phi) \cdot \int_{X} \widehat{A}\left(X, \nabla^{X}\right) \wedge \omega_{f^{*}(E \Delta)},
\end{aligned}
$$

where $C$ is a nonzero constant. Recall from [APS2], [L2, p. 195] that

$$
\eta\left(L, s_{L}, f_{L}, g_{L}\right)(\phi)=\sum_{\gamma \in F} \frac{1}{N} \chi_{\phi}(\gamma) \eta(\gamma)
$$

where $N$ is the common dimension of the two representations $\phi_{0}, \phi_{1}$ of $F$ such that $\phi=\phi_{1}-\phi_{0}$, and the higher eta-invariant of the Dirac operator of $\left[L, s_{L}, f_{L}, g_{L}\right]$ associated to $\mathcal{V}_{f_{L}}$ (see (2.1)) is given by

$$
\widetilde{\eta}\left(\left[L, s_{L}, f_{L}, g_{L}\right]\right)=\sum_{\gamma \in F} \eta(\gamma) \gamma \in \mathbb{C} F .
$$

From Equations (5.1) and (5.3) one easily deduces that there exists a nontrivial conjugacy class $\langle x\rangle$ of $F$ such that: $\mathcal{S}_{\langle x\rangle}\left(\sum_{\gamma \in F} \eta(\gamma) \gamma\right) \neq 0$. Thus, from Equation (5.2) and the fact that $\int_{X} \widehat{A}\left(X, \nabla^{X}\right) \wedge \omega_{f^{*}(E \Delta)} \neq 0$ one obtains: $\left\langle\widetilde{\eta}\left(\left[L \times X, s_{L} \times\right.\right.\right.$ $\left.\left.\left.s_{X}, f_{L} \times f, g_{L} \times \frac{1}{\epsilon} g_{X}\right]\right) ; \mathcal{T}_{\langle x\rangle}\right\rangle \neq 0$. We now remark that $\left[L, s_{L}, f_{L}\right]$ is a torsion element in $\Omega_{m}^{\text {spin }}(B F)$; thus $\left[L \times X, s_{L} \times s_{X}, f_{L} \times f_{X}\right]$ is also a torsion element in $\Omega_{n}^{\text {spin }}(B(F \times \Delta))$. Summarizing all the hypothesis of Proposition 4.3/Remark 4 of the previous Section are fulfilled and the proposition follows.

We can give a simple sufficient condition for the existence of a manifold $X$ as in the statement of the above two Propositions. We make a preliminary remark. Let $X$ be a closed spin manifold and $f: X \rightarrow B \Delta$ a continuous map; let $\omega_{f^{*}(E \Delta)} \in$ $\Omega^{*}(X) \widehat{\otimes} \bar{\Omega}_{*}(\mathbb{C} \Delta)$ be Lott's biform. By the work of Lott ([L1]) we know that

$$
\begin{aligned}
& \left\langle\int_{X} \widehat{A}\left(X, \nabla^{X}\right) \wedge \omega_{f^{*}(E \Delta)}, \tau_{c}\right\rangle \\
& \quad=\left\langle\left[\widehat{A}\left(X, \nabla^{X}\right)\right] \cup f^{*}[c] ;[X]\right\rangle, \quad \forall[c] \in H^{*}(\Delta, \mathbb{Q}) .
\end{aligned}
$$

This means that $X$ and $f: X \rightarrow B \Delta$ are such that $\int_{X} \widehat{A}\left(X, \nabla^{X}\right) \wedge \omega_{f^{*}(E \Delta)} \neq 0$ provided there exists at least one higher $\widehat{A}$-genus of $X$ with respect to $f$ which is 
different from 0 . The next Lemma gives a sufficient condition for the existence of such a manifold.

LEMMA 5.2. Let $n, m \in \mathbb{N}$. We assume that there $\exists k \in \mathbb{N}$, and $[c] \neq 0 \in$ $H^{(n-m)-4 k}(\Delta ; \mathbb{C})$. Then there exists a closed spin manifold of dimension $n-m$, $X$, and a continuous map $f: X \rightarrow B \Delta$ such that $\int_{X} \widehat{A}\left(X, \nabla^{X}\right) \wedge \omega_{f^{*}(E \Delta)} \neq 0$.

Proof. We first recall a fundamental result in bordism theory. For any integer $k \geqslant 0$ we denote by $\left\{i_{1}, i_{2}, \ldots, i_{r}\right\}$ a partition of $k$ (see [Mi-Sta]). One can define a natural map $\chi$

$$
\Omega_{n}^{\mathrm{spin}}(B \Delta) \otimes_{\mathbb{Z}} \mathbb{Q} \longrightarrow \oplus_{k} \geqslant 0 \oplus_{\left\{i_{1}, i_{2}, \ldots, i_{r}\right\}} H_{n-4\left(i_{1}+\cdots+i_{r}\right)}(B \Delta, \mathbb{Q})
$$

by

$$
\chi:[N, s, f] \longrightarrow \oplus_{k \geqslant 0} \oplus_{\left\{i_{1}, i_{2}, \ldots, i_{r}\right\}} f_{*}\left(P D\left(p_{i_{1}}(N) \wedge \cdots p_{i_{r}}(N)\right)\right),
$$

with $P D$ denoting Poincaré duality and $\left\{p_{j}(N)\right\}$ denoting the Pontryagin classes of $N$. It is shown in [Co-Fl] that this map is an isomorphism: that the two spaces are isomorphic follows from Theorem (14.2) p. 41 in [Co-Fl]. That the map $\chi$ implements this isomorphism follows from Theorem (17.4) p. 48 in [Co-Fl]. Notice that in $[\mathrm{Co}-\mathrm{Fl}]$ these results are established for the oriented bordism groups; as we are tensoring with the rationals the same results hold for the spin bordism groups (see [Sto, Chapter XI, p. 336]). Recall now that the $\widehat{A}$-genus may be written as: $\widehat{A}=1+\widehat{A}_{1}\left(p_{1}\right)+\widehat{A}_{2}\left(p_{1}, p_{2}\right) \cdots+\widehat{A}_{k}\left(p_{1}, p_{2}, \ldots, p_{k}\right)+\cdots$.

Let us fix a partition $\left\{i_{1}, i_{2}, \ldots, i_{r}\right\}$ of $k$ such that the coefficient of $p_{i_{1}} \cdots p_{i_{r}}$ in $\widehat{A}_{k}\left(p_{1}, p_{2}, \ldots, p_{k}\right)$ is not zero. Let us fix $\delta \in H_{n-m-4 k}(B \Delta, \mathbb{Q})$ such that $\langle\delta ;[c]\rangle \neq 0$. As the above map (5.5) is an isomorphism with $n-m$ in place of $n$, there exists

$$
\sum_{j=1}^{N} d_{j}\left[M_{j}, s_{j}, f_{j}\right] \in \Omega_{n-m}^{\mathrm{spin}}(B \Delta) \otimes_{\mathbb{Z}} \mathbb{Q},
$$

where the $d_{j} \in \mathbb{Q}$, such that

$$
\sum_{j=1}^{N} d_{j} f_{j_{*}}\left(P D\left(p_{i_{1}}\left(M_{j}\right) \wedge \ldots p_{i_{r}}\left(M_{j}\right)\right)\right)=\delta
$$

and such that for any other partition $\left\{j_{1}, j_{2}, \ldots, j_{\ell}\right\}$ of $k$ :

$$
\sum_{j=1}^{N} d_{j} f_{j_{*}}\left(P D\left(p_{j_{1}}\left(M_{j}\right) \wedge \ldots p_{j_{\ell}}\left(M_{j}\right)\right)\right)=0 .
$$

This implies that

$$
\left\langle\sum_{j=1}^{N} d_{j} f_{j_{*}}\left(P D\left(\widehat{A}_{k}\left(M_{j}\right)\right)\right) ;[c]\right\rangle \neq 0
$$


and so there exists $j \in\{1, \ldots, N\}$ such that

$$
\left\langle f_{j_{*}}\left(P D\left(\widehat{A}_{k}\left(M_{j}\right)\right)\right) ;[c]\right\rangle=\left\langle\widehat{A}\left(M_{j}\right) \cup f_{j}^{*}[c] ;\left[M_{j}\right]\right\rangle \neq 0 .
$$

Using (5.4), one then gets immediately the Lemma.

Proof of Theorem 0.1. It is a direct application of Proposition 5.1 and Lemma 5.2. Notice that in Proposition 5.1 we implicitly use the product formula for the higher eta-invariant (see §2); in that formula, which involves heat kernels, Lott's biform takes value in the noncommutative differential forms of the ConnesMoscovici algebra of $\Delta$ (thus not in the noncommutative forms of the group algebra of $\Delta$ ). This means that we can only apply the above Lemma to those groups for which the cyclic cocycle $\tau_{c}$ extends from the group algebra $\mathbb{C} \Delta$ to the ConnesMoscovici algebra $\mathcal{B}_{\Delta}^{\infty}$; this explains why we still have to assume that $\Delta$ is virtually nilpotent or Gromov hyperbolic in Theorem 0.1.

\section{Remarks on Concordance Classes}

(1) Let $N$ be a closed connected $n$-dimensional spin manifold which admits a metric of positive scalar curvature. So far we have been interested in nonbordant metrics. Suppose now that we relax our way of distinguishing PSC metrics and look for nonconcordant metrics. Our interest now lies in the set of concordance classes of PSC metrics, denoted $\tilde{\pi}_{0}\left(\mathcal{R}^{+}(N)\right)$. Stolz has proved in [St2] that there is a bijection between the set $\tilde{\pi}_{0}\left(\mathcal{R}^{+}(N)\right)$ and $R_{n+1}^{\text {spin }}(B \Gamma)$, with $\Gamma=\pi_{1}(M)$. This means that if we wish to compute the cardinality of the set of concordance classes of PSC metrics we can as well, and more generally, compute the cardinality of $R_{n+1}^{\text {spin }}(B \Gamma)$. As pointed out to us by Wolfgang Lück, the functorial properties of the relative bordism groups can sometimes be used in order to prove that $R_{*}^{\mathrm{spin}}(B \Gamma)$ is of infinite cardinality. For example: if $\Gamma_{1}$ and $\Gamma_{2}$ are two finitely generated groups and $\left|R_{n}^{\text {spin }}\left(\Gamma_{2}\right)\right|=\infty$, then

$$
\left|R_{n}^{\mathrm{spin}}\left(\Gamma_{1} * \Gamma_{2}\right)\right|=\left|R_{n}^{\mathrm{spin}}\left(\Gamma_{1} \times \Gamma_{2}\right)\right|=\left|R_{n}^{\mathrm{spin}}\left(\Gamma_{1} \rtimes \Gamma_{2}\right)\right|=\infty .
$$

The easy proof is left to the reader.

(2) Since, by functoriality, $R_{n}^{\text {spin }}(B\{1\})$ can be identified with a subgroup of $R_{n}^{\text {spin }}(B \Gamma)$, we see that for any discrete group $\Gamma$ and any $k \geqslant 2$ we have $\left|R_{4 k}^{\text {spin }}(B \Gamma)\right|$ $=\infty$. Indeed, the results in [LM, p. 329] imply that $\left|R_{4 k}^{\mathrm{spin}}(B\{1\})\right|=\infty$.

(3) In the same theme we also observe that our main result implies the following: Let $n \in \mathbb{N}, n \geqslant 7$. Let $F, \Gamma$ and $\Delta$ as in Theorem 0.1 . Then $\left|R_{n+1}^{\mathrm{spin}}(B(\Gamma \times \Delta))\right|=$ $+\infty$. Let us prove this claim. It is well known [Ros, p. 343] that one can find a closed connected spin manifold $Y$ of dimension $n-2 \geqslant 5$ such that $\pi_{1}(Y)=\Gamma \times \Delta$. We observe that the spin manifold $N:=Y \times S^{2}$ has dimension $n$, admits a PSC metric $g$ (since $S^{2}$ does) and has $\Gamma \times \Delta$ as fundamental group; we denote by $f: N \rightarrow B(\Gamma \times \Delta)$ the classifying map of the universal covering of $N$ and by 
$p: \Gamma \rightarrow F$ the surjective group homomorphism associated with the decomposition: $\Gamma=\Gamma^{\prime} \rtimes F$. Then, Lemma 5.2 and the proofs of Propositions 5.1 and 4.3 show that $N:=Y \times S^{2}$ admits an infinite number of nonbordant and thus nonconcordant metrics $g_{k}(k \in \mathbb{N})$ of PSC. Applying Stolz's result we conclude the proof.

(4) Always in the context of nonconcordant metrics of PSC we observe that one use of the map ${ }^{b} \alpha$ in Section 3 is the definition of a higher Gromov-Lawson index associated to a pair of PSC metrics on a closed spin manifold $N$ of dimension $n$. Let $g_{0}$ and $g_{1}$ two metrics of PSC on $N$; let $f: N \rightarrow B \Gamma$ be a classifying map and $s$ a spin structure on $N$. We consider the quadruple $\left[N \times[0,1], s, f, g_{0} \bigsqcup g_{1}\right] \in$ $R_{n}^{\text {spin }}(B \Gamma)$. We define the higher Gromov-Lawson index associated to the pair $\left(g_{0}, g_{1}\right)$ and to the triple $(N, s, f)$ as the noncommutative de Rham class

$$
\mathbf{h i}\left(N, s, f ; g_{0}, g_{1}\right):={ }^{b} \alpha\left(\left[N \times[0,1], s, f, g_{0} \coprod g_{1}\right]\right) \in \overline{\mathrm{H}}_{\text {even/odd }}\left(\mathcal{B}_{\Gamma}^{\infty}\right) .
$$

As a simple consequence of the Lichnerowicz formula we see that if $g_{0}, g_{1} \in$ $\mathcal{R}^{+}(N)$ and if $g_{0}$ is concordant to $g_{1}$ then, for each spin structure $s$ and each map $f: N \rightarrow B \Gamma$ one has hi( $\left.N, s, f ; g_{0}, g_{1}\right)=0$. Needless to say, there might be situations where the usual Gromov-Lawson index is zero but the whole higher Gromov-Lawson index is not. If the group is Gromov hyperbolic or virtually nilpotent then we get higher Gromov-Lawson indices hi $\left(N, s, f ; g_{0}, g_{1} ;[c]\right) \in \mathbb{C}$, with $[c] \in H^{*}(\Gamma, \mathbb{Q})$, by pairing with the corresponding (extendable) cyclic cocycle $\tau_{c} \in Z C^{*}(\mathbb{C} \Gamma)$.

(5) Let $g_{0}$ and $g_{1}$ be PSC metrics on $N$ having a nonzero higher GromovLawson index. Let $X$ be a closed manifold, $\Gamma$ a discrete group and $f: X \rightarrow B \Gamma$ a continuous map. We assume that $\Gamma$ is virtually nilpotent or Gromov hyperbolic and that there exists a nonzero $\widehat{A}$-roof genus of $X$ with respect to $f$. Then, if $\epsilon$ is small enough, the two product PSC metrics $g_{0} \times 1 / \epsilon g_{X}$ and $g_{1} \times 1 / \epsilon g_{X}$ remain nonconcordant. Indeed, using Proposition 2.1 one can show that these two product metrics have a nonzero higher Gromov-Lawson invariant. This remark gives a partial answer to a question raised in [We].

(6) The higher Gromov-Lawson index fits into the following relative index formula: let $\left(M, s, f, g_{\partial M}\right)$ be a representative of an element in $R_{n}^{\mathrm{spin}}(B \Gamma)$. Let $g_{0}, g_{1} \in \mathcal{R}^{+}(\partial M)$. Then

$$
\begin{aligned}
& { }^{b} \alpha\left[M, s, f, g_{0}\right]-{ }^{b} \alpha\left[M, s, f, g_{1}\right] \\
& \quad=\mathbf{h i}\left(\partial M,\left.s\right|_{\partial M},\left.f\right|_{\partial M} ; g_{1}, g_{0}\right) \text { in } \bar{H}_{*}\left(\mathcal{B}_{\Gamma}^{\infty}\right) .
\end{aligned}
$$

In order to prove this formula let $G_{0}$ and $G_{1}$ be two metrics on $M$ extending $g_{0}$ and $g_{1}$, respectively. Let $G_{\text {cyl }}$ be a metric on $\partial M \times[0,1]$ extending $g_{0} \bigsqcup g_{1}$. We consider the double $Z=M \cup_{\partial M}(\partial M \times[0,1]) \cup_{\partial M}(-M)$. We endow $Z$ with the metric $G_{Z}$ obtained by gluing $G_{0}, G_{\text {cyl }}$ and $G_{1}$. The manifold $Z$ inherits in a natural way a spin structure $s_{Z}$ and a classifying map $f_{Z}$. The index class associated to the Dirac operator defined by these data is zero (being a double, $\left(Z, s_{Z}, f_{Z}\right)$ is 
the boundary of a triple $\left.\left(W, s_{W}, f_{W}\right)\right)$. Then, by applying the additivity formula for index classe we obtain

$$
\begin{aligned}
0= & { }^{b} \Theta\left[M, s, f, g_{0}\right]+{ }^{b} \Theta\left[\partial M \times[0,1], s_{\partial M},\left.f\right|_{\partial M}, G_{\text {cyl }}\right]- \\
& -{ }^{b} \Theta\left[M, s, f, g_{1}\right] .
\end{aligned}
$$

Applying the Chern character we immediately get (6.1).

The higher Gromov-Lawson index is in fact a higher noncommutative spectral flow, see [DZ2, Wu1, LP4]; formula (6.1) can also be proved by using a variational formula for $b$-index classes. This formula is proved in [DZ2, §5 b)] in the family case and in [LP4, Proposition 8] in the noncommutative case.

\section{Acknowledgements}

Part of this work was done while Eric Leichtnam was visiting University of Münster. He would like to thank Wolfgang Lück and Michael Joachim for helpful conversations. We are grateful to John Lott for very interesting discussions and for suggesting Lemma 5.2. Finally, we thank Thomas Schick and the anonymous referee for helpful comments on earlier versions of this paper.

\section{References}

[APS 2,3] Atiyah, M. F., Patodi, V. and Singer, I.: Spectral asymmetry and Riemannian geometry II, Math. Proc. Cambridge Philos. Soc. 78 (1975), 405; III: 79, (1975), 71.

[AS V] Atiyah, M. F. and Singer, I.: The index of elliptic operators V, Ann. Math. 93 (1971), 139.

[Bo-Gi] Botvinnik, B. and Gilkey, P.: The eta-invariant and metrics of positive scalar curvature, Math. Ann. 302 (1995), 507.

[Bu] Bunke, U.: A $K$-theoretic relative index theorem and Callias-type Dirac operators, Math. Ann. 303 (1995), 241.

[Bur] Burghelea, D.: The cyclic homology of the group rings, Comment Math. Helv. 60 (1985), 354.

[Co-Fl] Conner, P. and Floyd, E.: Differentiable Periodic Maps, Springer, New York, 1964.

[Co] Connes, A.: Non-commutative differential geometry, Publ. IHES 62 (19xx), 41.

[DZ1] Dai, X. and Zhang, W.: Splitting of the family index, Comm. Math. Phys. 182 (1996), 303.

[DZ2] Dai, X. and Zhang, W.: Higher spectral flow, J. Funct. Anal. 157 (1998), 432.

[G-L] Gromov, M. and Lawson, B.: Positive scalar curvature and the Dirac operator on complete Riemannian manifolds, Publ. Math. IHES 58 (1983), 83.

[Ha] Hajduk, B.: On the obstruction group to the existence of Riemannian metrics of positive scalar curvature, In: Proc. International Conf. on Algebraic Topology, Poznan, Lecture Notes in Math. 1481, Springer, New York, 1991, p. 63. p. 62.

[Ka] Karoubi, M.: Homologie cyclique et $K$-théorie, Astérisque 149 (1987).

[KS] Kreck, M. and Stolz, S.: Nonconnected moduli spaces of positive sectional curvature metrics, J. Amer. Math. Soc. 6(4) (1993), 825.

[LM] Lawson, B. and Michelson, M.-L.: Spin Geometry, Princeton Math. Ser. 38, Princeton Univ. Press, 1989. 
[LLP] Leichtnam, E., Lott, J. and Piazza, P.: On the homotopy invariance of higher signatures on manifolds with boundary, J. Differential Geom. 54 (2000), 000-000.

[LP1] Leichtnam, E. and Piazza, P.: The $b$-pseudodifferential calculus on Galois coverings and a higher Atiyah-Patodi-Singer index theorem, Mém. Soc. Math. France 68 (1997).

[LP2] Leichtnam, E. and Piazza, P.: Spectral sections and higher Atiyah-Patodi-Singer index theory on Galois coverings, Geom. Funct. Anal. 8 (1998), 17.

[LP3] Leichtnam, E. and Piazza, P.: Homotopy invariance of twisted higher signatures on manifolds with boundary, Bull. Soc. Math. France 127 (1999), 307.

[LP4] Leichtnam, E. and Piazza, P.: Dirac index-classes and the noncommutative spectral flow, Preprint MSRI No. 2001-017, http://www.msri.org/publications/preprints/ index.html

[L1] Lott, J.: Superconnections and higher index theory, Geom. Funct. Anal. 2 (1992), 421.

[L2] Lott, J.: Higher eta-invariants, K-Theory 6 (1992), 191.

[L3] Lott, J.: Diffeomorphisms, analytic torsion and non commutative geometry, Mem. Amer. Math. Soc. 141(673) (1997).

[Me] Melrose, R.: The Atiyah-Patodi-Singer Index Theorem. A. and K. Peters, Wellesley, MA, 1993.

[MP] Melrose, R. and Piazza, P.: An index theorem for families of Dirac operators on odd dimensional manifolds with boundary, J. Differential. Geom. 46(2) (1997), 287.

[Mi-Sta] Milnor, J. and Stasheff, J.: Characteristic classes, Ann. of Math. Stud. 76, Princeton Univ. Press, 1974.

[Ros] Rosenberg, J.: $C^{*}$-algebras, positive scalar curvature, and the Novikov conjecture II, In: Geometric Methods in Operator Algebras, Pitman Res. Notes, 123, Pitman, London, 1986, p. 341.

[St1] Stolz, S.: Positive scalar curvature metrics. Existence and classification questions, In: Proc. of ICM Zurich 1994, Birkhaüser-Verlag, Basel, 1995, p. 626.

[St2] Stolz, S.: Concordance classes of positive scalar curvature metrics, Preprint, 1999 (see http: / / www.nd.edu/stolz/preprint.html).

[Sto] Stong, R.: Notes on Cobordism Theory, Princeton Univ Press, 1968.

[We] Weinberger, S.: Higher $\rho$-invariants, In: M. Farber, W. Lück and S. Weinberger (eds), Tel Aviv Conference, Rothenberg Festschrift, Contemp. Math. 231, Amer. Math. Soc., Providence, 1999.

[Wu1] Wu, F.: The noncommutative spectral flow, Unpublished, 1997.

[Wu2] Wu, F.: The higher $\Gamma$-index for coverings of manifolds with boundaries, In: Cyclic Cohomology and Noncommutative Geometry, Fields Inst. Comm. 17, Amer. Math. Soc., Providence, 1997, p. 169. 